\title{
Design and performance analysis of an Integrated Starter-Alternator for Hybrid Electric Vehicles
}

\author{
Florin Nicolae Jurca, Radu Petru Hangiu, Claudia Marțiş \\ Technical University of Cluj-Napoca, Romania \\ florin.jurca@mae.utcluj.ro
}

Keywords: machine design, permanent synchronous machine, hybrid vehicle

\begin{abstract}
In automotive applications, the requirements for the electrical machines are about performance and reliability - the permanent magnet synchronous machines ensure these requirements are fulfilled. The approach of the present paper is to analyze the permanent synchronous machines suited for the integrated starter-alternator (ISA) applications. The study design requires some analytical, followed by a numerical analysis and also a finite element method in order to achieve the performances of the machine. The operation modes of an ISA system and the constraints for an electric machine acting as an ISA are detailed. Finally a model for a ISA hybrid vehicle, developed in AMESim is presented and the results of a simulated drive cycle are presented.
\end{abstract}

\section{Introduction}

The ISA is an electric machine that combines the functionalities of the starter and of the alternator found in conventional systems driven by an internal combustion engine (ICE). In the case of road vehicles, using a single machine with a two quadrant operation reduces the weight of the system and allows for implementing new functionalities that reduce fuel consumption and increase ride comfort and vehicle performance: start/stop functionality, ICE assistance (power boost) at high loads, reactive braking, active synchronization of engine and transmission, continuous tractive force to the wheels when using automatic shift transmissions, torsional vibration damping, active crankshaft torque ripple cancellation (active flywheel).

The operating conditions of the ISA are required in the starter mode so the torque needed for the starting of the ICE can be developed at low speed. In the generator mode, ISA should produce the required power on a wide range of speeds, usually between 1000 and $6000 \mathrm{rpm}$. [1], [3].

ISA can be connected with gasoline or diesel engine, either directly trough the crankshaft or indirectly trough the belt system; based on that, the systems are called ,belt-driven alternator starter“(BAS) and „normal ISA“, respectively.

Comparative whit other types of electrical machines, the permanent magnet (PM) synchronous machines have some important advantages like high power density, high efficiency and the possibility to work in high overload. From the efficiency point of view, the PM machine with 8 to12 poles is preferable. The encased rotor, which can protect permanent magnet from iron dregs in the cooling air and the NdFeB material from corrosion, is preferred; [2].

An ISA is basically a mild hybrid electric vehicle that is capable of providing $20 \%-25 \%$ fuel savings, as claimed in [3]. The ISA is expected to not only meet the increasing vehicle electrical load demand but also address the challenges of fuel economy, efficiency, and emission.

The present paper approaches a preliminary design is presented and the results are in FLUX 2D and Simulink in order to obtain the performances of the machine. The operation modes of an ISA system and the constraints for an electric machine acting as an ISA are detailed. Finally a model for a ISA hybrid vehicle, developed in AMESim is presented and the results of a simulated drive cycle are presented. 


\section{Preliminary design}

An electrical machine design problem is to find a set consisting of topological structure, materials, and geometry for a specific application. The selection of the proper machine topology for a specific application is a difficult problem to be solved during the design process.

In order to improve the electrical machines performances, several winding topologies will be analyzed. The output performances of the studied motor are: $P-6.5(\mathrm{~kW})$; rated voltage $U_{\mathrm{n}}-72$ $(\mathrm{V})$; rated speed $n_{n}-400 \mathrm{rpm}$; pole pair number $p-15$.

The output power of the electric machine, when the leakage reactance is neglected, is expressed by the number of phases of the machine, $n_{p h}$, the phase current, $i(t)$, the inducted electromotive force (emf), $e(t)[7]$ :

$$
P_{\text {out }}=\eta \cdot \frac{n_{p h}}{T} \cdot \int_{0}^{T} e(t) \cdot i(t) d t=\eta \cdot n_{p h} \cdot k_{p} \cdot E_{\max } \cdot I_{\max }
$$

In the previous equation, $T$ is the period of one cycle of emf, $E_{\max }$, and $I_{\max }$ represent the peak value of the emf and phase current, $\eta$ is the estimated efficiency, ke-the electromotive force coefficient.

$$
E_{\max }=k_{E} \cdot N_{t} \cdot B_{\text {gap }} \cdot D_{\text {gap }} \cdot L_{m} \cdot \frac{f_{s}}{p}
$$

Using the coefficients $k_{L}=L_{m} / D_{\text {gap }}$ (geometric coefficient), $k_{i}=I_{\max } / I_{r m s}$ (current coefficient) and the phase load ampere-turns [7],

$$
A_{t}=\frac{2}{\pi} \cdot N_{t} \cdot \frac{I_{r m s}}{D_{g a p}}
$$

it is possible to define the air-gap diameter of the machine:

$$
D_{\text {gap }}=\sqrt[3]{\frac{2 \cdot p \cdot P_{\text {out }}}{\pi \cdot n_{p h} \cdot A_{t} \cdot k_{e} \cdot k_{i} \cdot k_{p} \cdot k_{L} \cdot \eta \cdot B_{g a p} \cdot f_{s}}}
$$

The power and the voltage coefficients are defined according to the type and current wave form, in this case the sinusoidal wave form is $\mathrm{k}_{\mathrm{i}}=\sqrt{2}, \mathrm{k}_{\mathrm{p}}=0.5$ [4], [7].

By choosing the type of permanent magnet and the number of slots, and also using the expression of the air-gap diameter, the designer can determine all other geometrical parameters.

The air-gap flux density is computed based on the following formula:

$$
B_{\text {gap }}=\frac{h_{m} \cdot B_{r m}}{\frac{D_{g a p}}{2} \cdot\left(\ln \left(\frac{R_{s c r}-g a p}{R_{s o}}\right)+\ln \left(\frac{R_{c r}}{R_{c r}-g a p}\right)\right)}
$$

For active parts of the machine, it was used good quality PMs material, of Nd-Fe-B N48 type, with 1.4 T remanent flux density. The steel is made of M530-50A sheets.

For motor regime operation of PMSM (with magnetic anisotropy), one can use the typical load phasor diagram, in $d-q$ reference frame, Fig 1 . From this phasor diagram one will get the $d-q$ axis reactance's equations, function of phase voltage, $U_{\mathrm{ph}}$, phase electromotive force, $E_{\mathrm{ph}}$, phase resistance, $R_{\mathrm{ph}}, d-q$ axis currents and internal angle, $\delta[7]$ : 


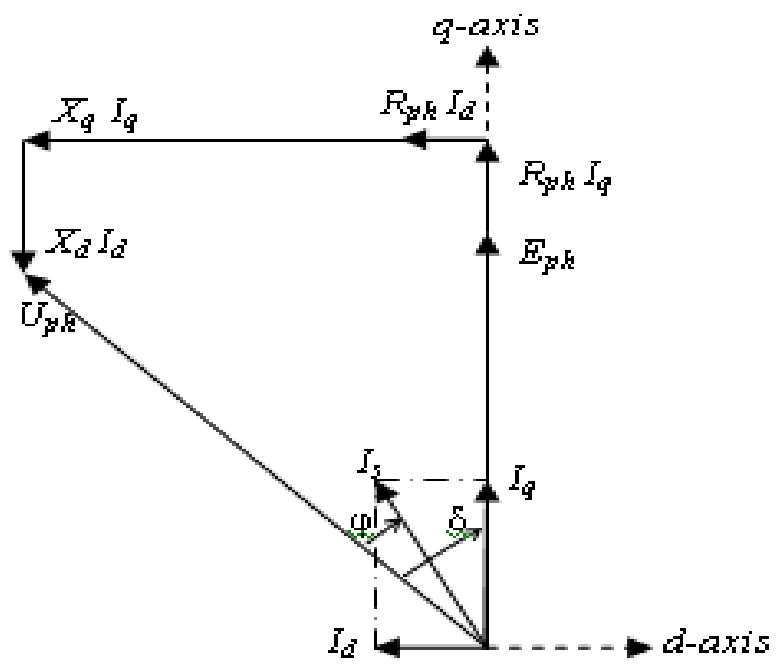

Fig. 1. Phasor diagram for PMSM in motor-load operating [7].

$$
\left\{\begin{array}{l}
X_{d}=\left(U_{p h} \cdot \cos (\delta)-E-R_{p h} \cdot I_{q}\right) / I_{d} \\
X_{q}=\left(U_{p h} \cdot \sin (\delta)+R_{p h} \cdot I_{d}\right) / I_{q}
\end{array}\right.
$$

Also, by using the phasor diagram, it is possible to compute the source current, $\mathrm{I}_{\mathrm{s}}=\sqrt{\mathrm{I}_{\mathrm{d}}{ }^{2}+\mathrm{I}_{\mathrm{q}}{ }^{2}}$, where $I_{d}$ and $I_{q}$ are obtained by using the following formulas:

$$
\left\{\begin{array}{l}
I_{d}=\frac{U_{p h} \cdot\left(X_{q} \cdot \cos (\delta)-R_{p h} \cdot \sin (\delta)-E_{p h} \cdot X_{q}\right.}{R_{p h}^{2}+X_{d} \cdot X q} \\
I_{q}=\frac{U_{p h} \cdot\left(X_{d} \cdot \cos (\delta)+R_{p h} \cdot \sin (\delta)-E_{p h} \cdot R_{p h}\right.}{R_{p h}^{2}+X_{d} \cdot X_{q}}
\end{array}\right.
$$

The frequency, the number of turns, the air-gap flux per pole and a demagnetization coefficient (usually between $0.8-0.9$ for rare earth PMs) make up the expression of the electromotive force:

$$
E_{p h}=\sqrt{2} \cdot \pi \cdot f_{s} \cdot N_{t} \cdot k_{w s} \cdot \Psi_{g a p} \cdot k_{d}
$$

Next, the usual electromechanical characteristics can also be computed [4]:

$$
\begin{aligned}
P_{\text {in }} & =n_{p h} \cdot U_{p h} \cdot\left(I_{q} \cdot \cos (\delta)-I_{d} \cdot \sin (\delta)\right) \\
P_{\text {out }} & =P_{\text {in }}-\sum \text { Losses }
\end{aligned}
$$

$\mathrm{P}_{\text {out }}$ - the output power, function of input power and the sum of losses (iron, copper, mechanical and supplementary losses).

$$
\begin{gathered}
T_{m}=\frac{P_{\text {out }}}{\Omega} \\
\cos (\varphi)=\frac{P_{\text {in }}}{n_{p h} \cdot U_{p h} \cdot I_{s}} \\
\eta=\frac{P_{\text {in }}}{P_{\text {out }}}
\end{gathered}
$$




\section{Innovation for Sustainable Development}

The resulted main dimensions and the results for operation at rated point are shown in Table1.

Table I The obtained results for the designed electrical machine

\begin{tabular}{|l|c|}
\hline Stator outer diameter [m] & 0.186 \\
\hline Rotor inner diameter [m] & 0.201 \\
\hline Rotor outer diameter [m] & 0.230 \\
\hline Shaft diameter [m] & 0.130 \\
\hline Stack length [m] & 0.280 \\
\hline Air-gap [m] & 0.0015 \\
\hline Air-gap flux density [T] & 1 \\
\hline Rates speed [rot/min] & 400 \\
\hline Phase emf [V] & 42 \\
\hline Rated current [A] & 72 \\
\hline Power factor [\%] & 0.9 \\
\hline Efficiency [\%] & 0.87 \\
\hline Torque [N*m] & 150 \\
\hline Iron loss [W] & 212 \\
\hline
\end{tabular}

\section{Magnetic field analysis}

The finite element method (FEM) is a powerful tool for the design of the electrical machines and others electromagnetic devices. FEM is a simple, robust and efficient widely used method of obtaining a numerical approximate solution for a given mathematical model of the machine.

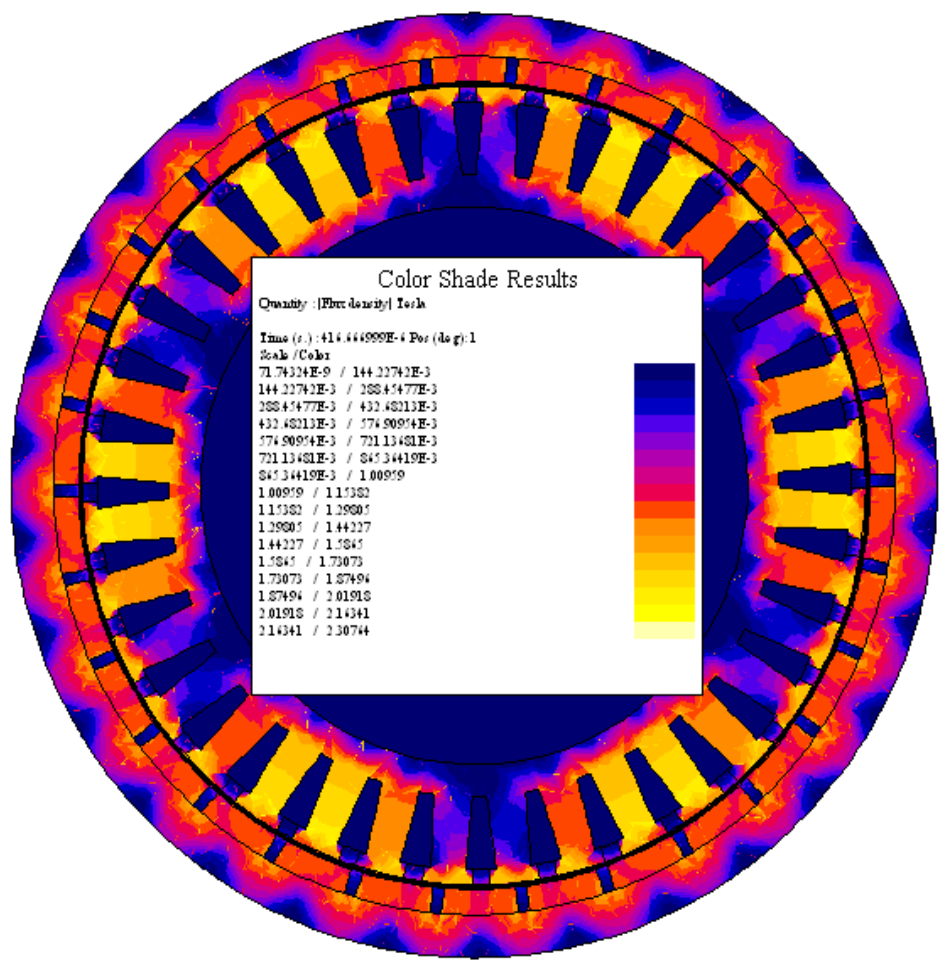

Fig.2. Magnetic flux density map

This analysis has been carried out using Flux2D software. The magnetic flux density map in the cross-section of the machine is presented in Fig. 2. 


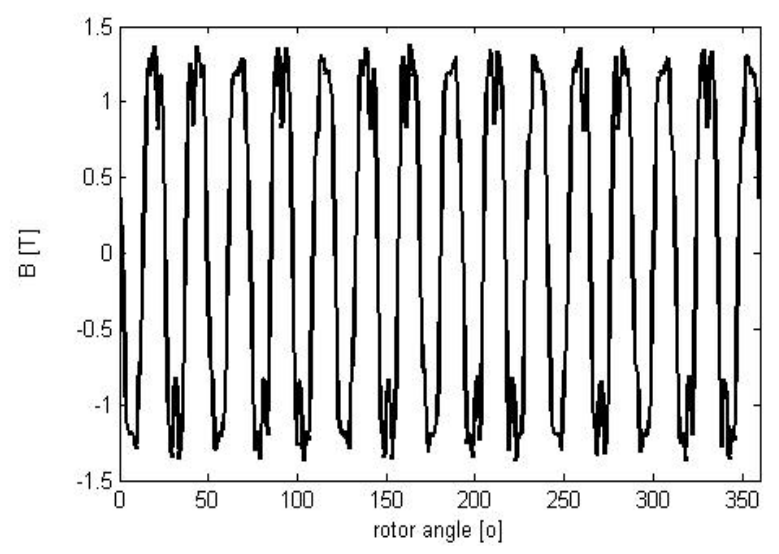

Fig.3. Air-gap magnetic flux density

The rms value of the emf induced in the stator windings of is given by:

$$
E_{r m s}=\frac{E_{\max }}{\sqrt{2}}
$$

With $\mathrm{E}_{\text {rms }}$ computed with Eq. (2), where

$$
B_{g_{-} a v}=\sqrt{\frac{1}{2 \pi} \int_{0}^{2 \pi} B_{g}{ }^{2}(\alpha) d \alpha}
$$

with $B_{g}(\alpha)$ the distribution of the magnetic field density along the air gap. For no-load condition, the air gap magnetic flux density distribution is depicted in Fig. 3, giving an average value of $1.02 \mathrm{~T}$, thus an rms value of the induced emf equal to $41.5 \mathrm{~V}$, for the rated driven speed.

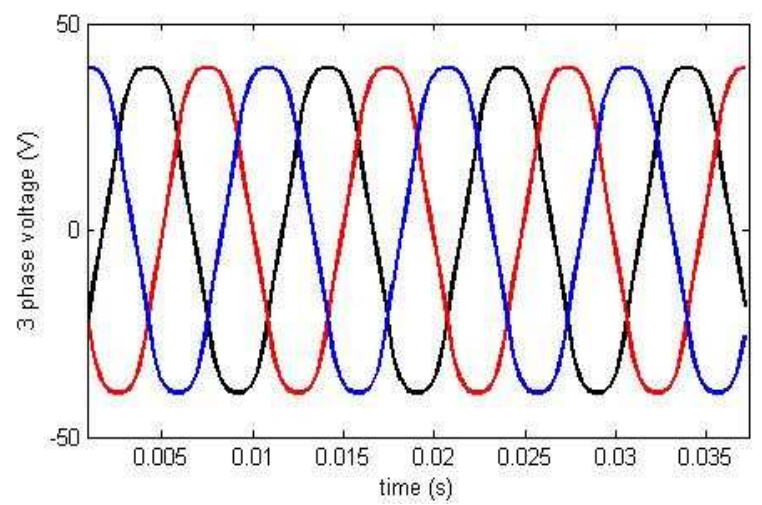

Fig.4. Three phase voltage obtained in no-load condition
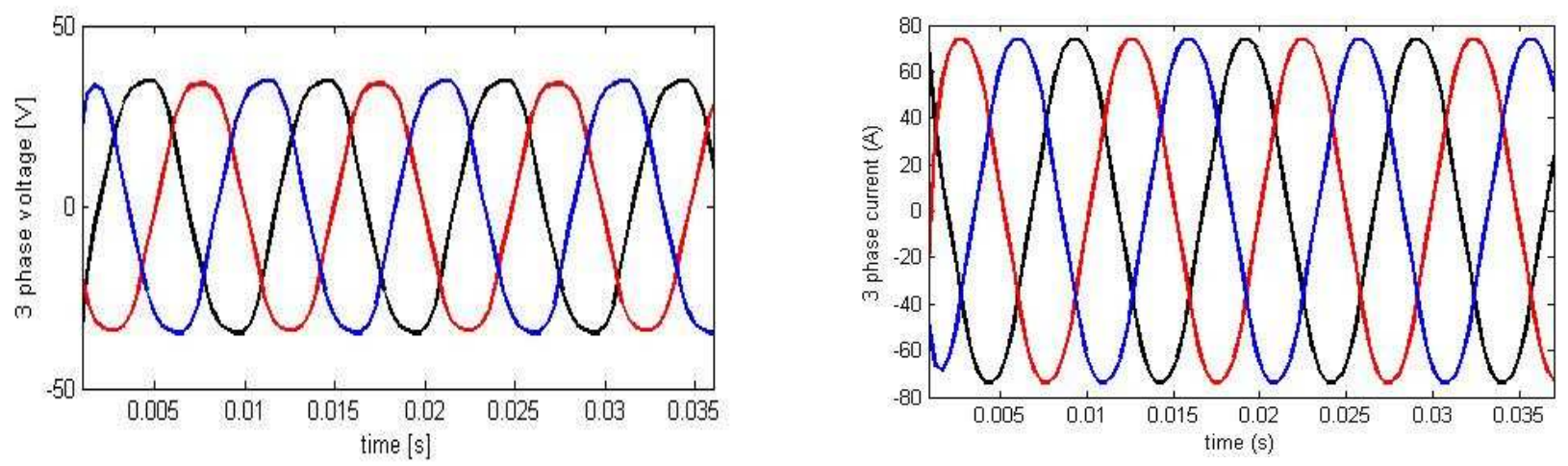

Fig.5. Three voltage and current obtained in load condition at $400 \mathrm{rpm}$. 


\section{Simulation of an ISA hybrid vehicle.}

The system model (Fig. 6) has been developed in AMESim, which is a multi-domain simulation software for the modeling and analysis of one-dimensional (1D) systems. It comprises discrete models of the ICE, gearbox, control system, electric loads, electric machine and power inverter, connected together to form a global model of a vehicle. Machine and the chosen control method is FOC with a maximum torque per ampere (MTPA) strategy for lower speeds followed by a flux weakening control for higher speeds. The torque reference is derived either from the torque required by the ECU during ICE cranking or from the DC bus regulator during generation [5], [6].

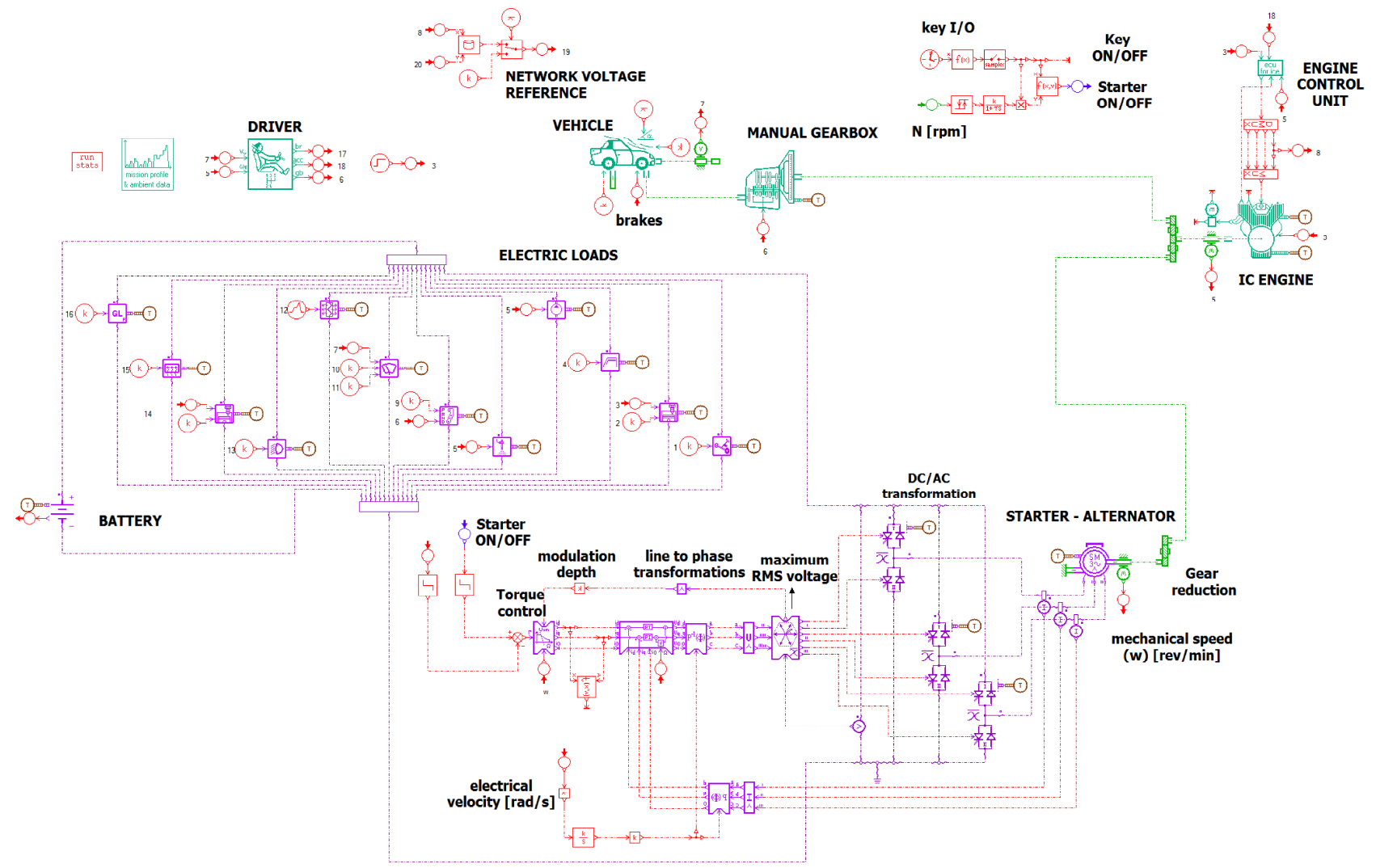

Fig. 6. ISA system model.

The simulation is carried out on a New European Driving Cycle (NEDC) that consists of four repeated ECE-15 driving cycles and an Extra-Urban driving cycle, and represents the typical car usage in Europe.

The electric machine chosen for the system is presented above in the paper. The internal combustion engine is a 2.0 liter spark ignition engine with a rated power output of $110 \mathrm{KW}$. DC bus voltage is set at $72 \mathrm{~V}$. Multiple electric loads are connected to the system ranging from windscreen wiper motors and power window motors to electric fuel pumps. The vehicle's total mass is estimated at $1.47 \mathrm{t}$ and its active aerodynamic drag area is $2 \mathrm{~m}^{2}$.

At the beginning of the drive cycle, the ICE is cranked by the ISA. Once the crankshaft reaches the speed necessary for the combustion process the ECU takes over the ICE's control and the ISA switches to generator mode. MTPA control strategy is used at engine cranking when due to the low speeds the system's voltage constraints are not exceeded.

The ISA output torque and current variation during the start-up process and immediately after is given in Fig. 7. The peak torque value is $125 \mathrm{Nm}$. The profile of engine speed is given in Fig. 8. 

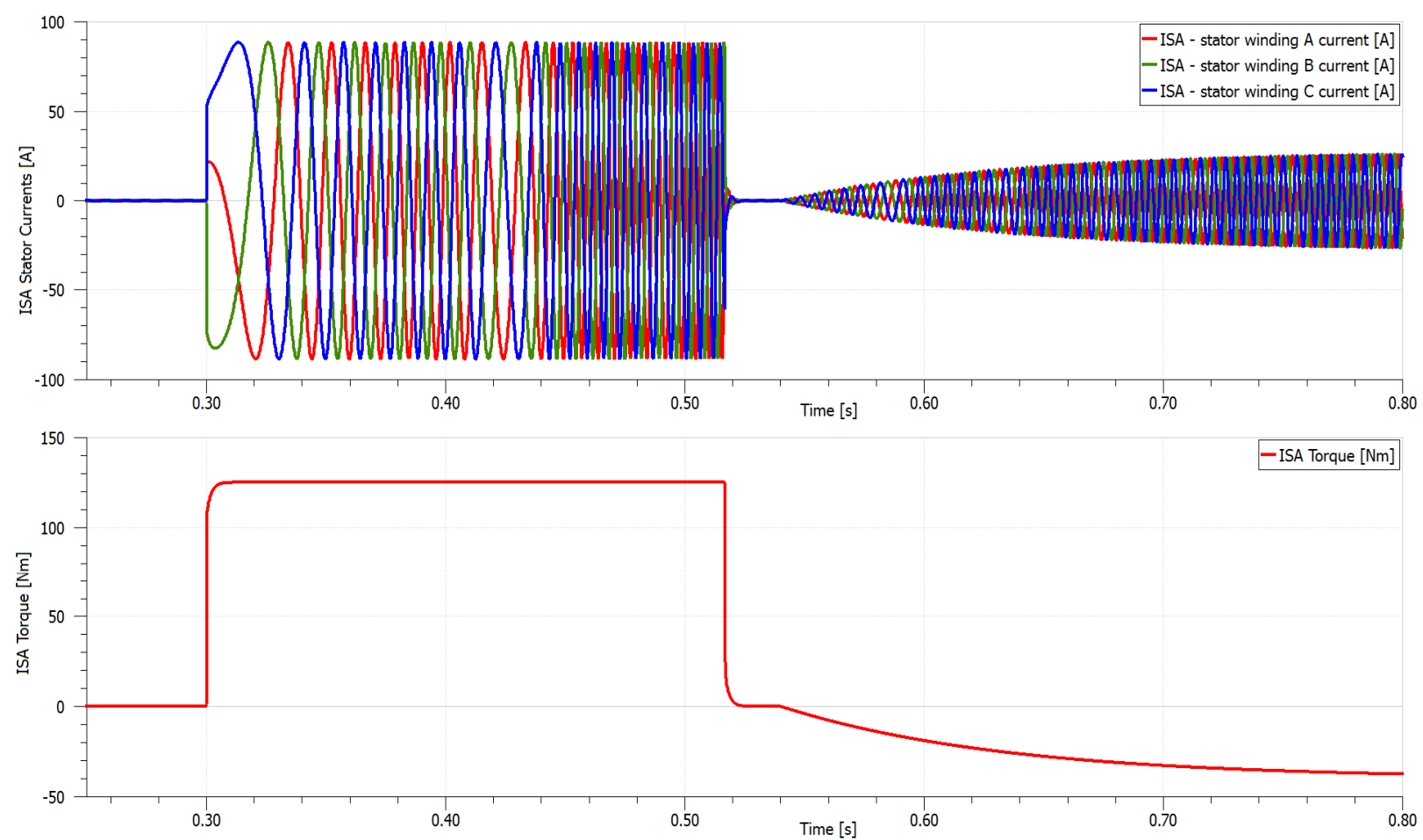

Fig. 7. ISA torque and current profile at start-up.

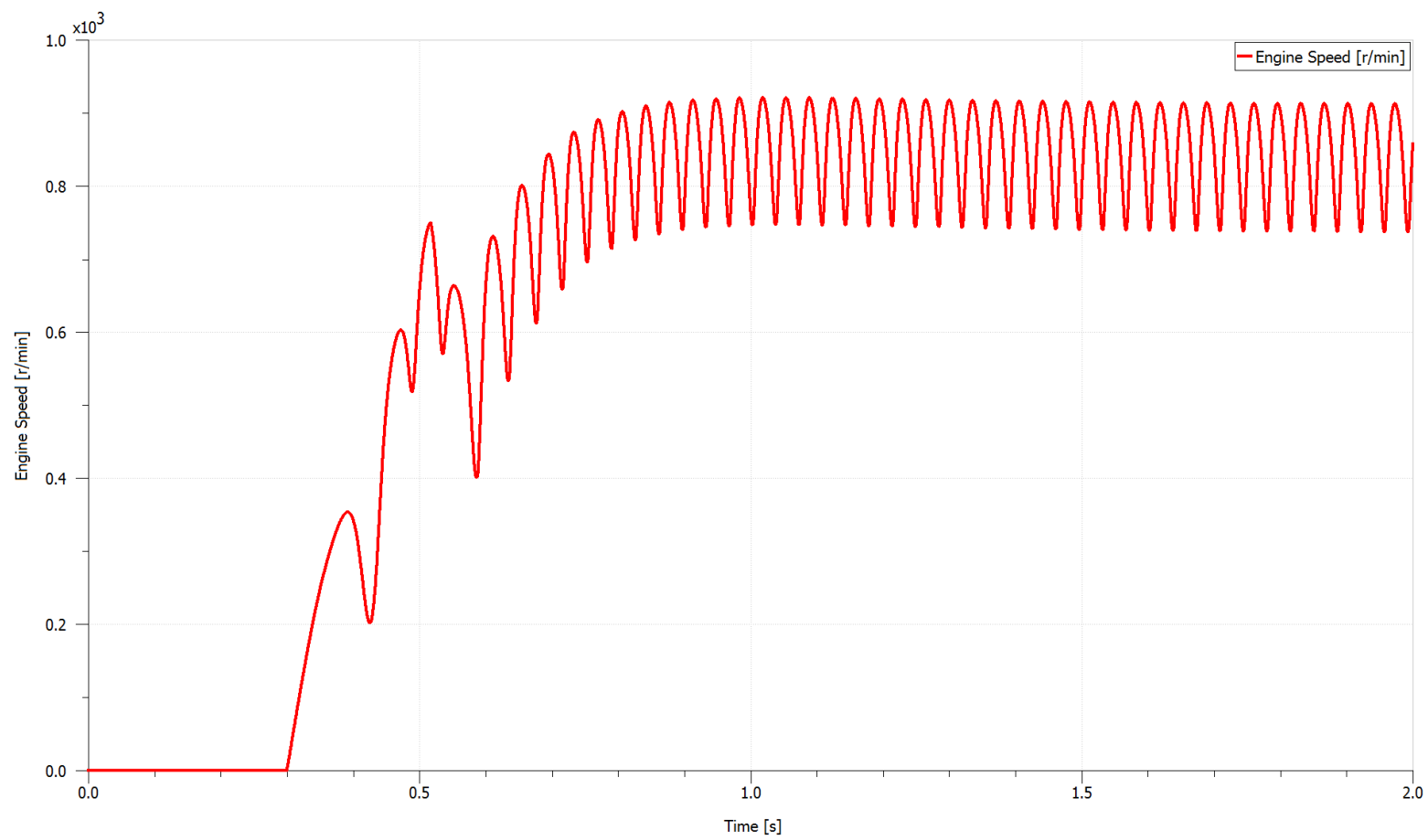

Fig. 8. Profile of the engine speed.

Total fuel consumption over the considered driving cycle was $933.63 \mathrm{~g}$ when the start-stop and regenerative braking strategies were not employed. By employing those strategies the fuel consumption was reduced to $800.71 \mathrm{~g}$ representing a fuel economy of $15 \%$. 


\section{Conclusions}

Replacing the starter and alternator in an ICE vehicle with a single unit that performs both functions is the next step in the automotive industry. The ISA allows for an easier implementation of simple fuel saving and emission reduction functionalities such as the start/stop functionality. With adequate control more complex hybrid operation modes can be used.

The paper presents the theoretical approach of a PM radial-flux machine with outer rotor, suitable for automotive application (integrated starter-alternator). The preliminary design model of the machine was developed, followed by a simulation carried on by implementing the topology in Flux 2D for generator regime. The ISA regime was developed in AMESIM programs in order to obtain the profiles of torque, currents and engine speed.

\section{Acknowledgment.}

This paper was supported by the project "Development and support of multidisciplinary postdoctoral programmers in major technical areas of national strategy of Research - Development Innovation" 4D-POSTDOC, contract no. POSDRU/89/1.5/S/52603, project co-funded by the European Social Fund through Sectoral Operational Programme Human Resources Development 2007-2013.

\section{References}

[1] W. Cai, Comparison and review of electrical machine for integrate starter-alternator applications,. Industry applications society annual meeting (IAS), IEEE 386-393 (2004).

[2] M. Barcaro, A. Alberti, L.Faggion, M. Sgarbossa, Dai Pr'e M, N. Binachi, S. Bologni, IPM machine drive design and tests for an integrated starter-alternator application. Industry applications society annual meeting (IAS), IEEE 1-8 (2008).

[3] M. Barcaro, A. Alberti, L.Faggion, M. Sgarbossa, Dai Pr'e M, N. Binachi, Expereimental tests on a 12-slot 8-pole integrated starter-alternator. Proceedings of the 2008 International Conference on Electrical Machines 1-6.

[4] D.Fodorean, A. Djerdir, I.A. Viorel, A. Miraoui, A Double Excited Synchronous Machine for Direct Drive Application - Design and Prototype Tests, IEEE Transactions on Energy Conversion, vol.22, n.3, pp.656-665, September, (2007).

[5] A. Emadi, S. S. Williamson, and A. Khaligh, Power electronics intensive solutions for advanced electric, hybrid electric, and fuel cell vehicular power systems, Conf. rec. IEEE 40th IAS Annu. Meeting, vol. 2, (2005) pp. 1015 - 1022,

[6] A. K. Jain, S. Mathapati, V. T. Ranganathan and V. Narayanan, Integrated starter generator for 42-v powernet using induction machine and direct torque control technique", IEEE Transactions On Power Electronics, Vol. 21, No. 3, May (2006), pp. 701-709.

[7] D. Fodorean, Global design and optimization of a permanent magnet synchronous machine used for light electric vehicle, in IntechWeb, Electric vehicles-modelling and simulations, Rijeka,(2011), pp 177-198 